\title{
Conhecimentos de profissionais de saúde sobre o princípio da atenção diferenciada aos povos indígenas
}

\author{
Health professionals' knowledge about the differentiated attention principles to indigenous \\ peoples
Conocimientos de profesionales de la salud sobre el principio de atención diferenciada a los pueblos indígenas

Ana Paula Barbosa Alves ${ }^{1 \star}$, Tercinara da Silva Aguiar ${ }^{1}$, Simone Lopes de Almeida ${ }^{1}$, Luiza Brum Argenta $^{1}$, Hosana Carolina dos Santos Barreto ${ }^{1}$, Marcos Antonio Braga de Freitas ${ }^{1}$.

\section{RESUMO}

Objetivo: Averiguar os conhecimentos de profissionais de saúde sobre o princípio da atenção diferenciada aos povos indígenas. Métodos: Trata-se de uma pesquisa exploratória de abordagem qualitativa. Como campo foi escolhido um hospital de um município do estado de Roraima. Foram aplicados questionários com 11 perguntas semiestruturados para 7 profissionais de saúde. Resultados: A maioria dos participantes não tinham experiência de trabalho com povos indígenas, e apresentaram uma compreensão limitada, não sabiam, ou não conseguiam construir uma opinião formada. Nas análises das falas dos profissionais, verificou-se que eles conhecem algumas características sobre atenção diferenciada, como por exemplo, percebem os aspectos culturais e a diversidade linguística e étnica dos povos indígenas. No entanto, não faziam relação desses aspectos, para embasar atitudes e comportamentos em suas práticas assistenciais em saúde. Conclusão: Diante da percepção dos profissionais de saúde, foram evidenciadas visões diferenciadas, sendo possível conhecer suas experiências em relação a realidade do trabalho no hospital, verificando uma preocupação com os conhecimentos sobre os povos indígenas e com a qualidade na atenção. As formações dos diferentes profissionais de saúde precisam ser revistas, no sentido de preparálos para o trabalho em contextos interculturais.

Palavras-chave: Saúde, Povos indígenas, Competência cultural.

\begin{abstract}
Objective: Check the health professionals' knowledge about the differentiated attention principles to indigenous peoples. Methods: Treats of an exploratory research of qualitative approach. As research area has been chosen a hospital in a city at Roraima's State. Has been applied 11 semi-structured questions in a quiz to 7 health professionals. Results: The most of professionals had not experience with indigenous peoples, and showed a limited comprehension, they did not know or did not know how to express their opinions. In the professionals' speech analysis, has been noticed that they know some characteristics about differentiated attention as they notice cultural aspects, linguistic and ethnic diversity of indigenous peoples. However, they did not relate these aspects to support their attitudes and behaviors at their health care practices. Conclusion: Were evidenced different prospects of the health professionals, being able to know their experiences about the daily reality at the hospital, were verified a concern about the knowledge about the indigenous peoples and attention quality. The different university graduations need to be checked on sense of prepare them to deal in different cultural contexts.
\end{abstract}

Keywords: Health, Indigenous peoples, Cultural competency.

1 Universidade Federal de Roraima (UFRR), Boa Vista - RR. *E-mail: paula.alves@ufrr.br

SUBMETIDO EM: 7/2020

ACEITO EM: 8/2020

PUBLICADO EM: 11/2020

REAS/EJCH | Vol.12(11) | e4631 | DOI: https://doi.org/10.25248/reas.e4631.2020 Página 1 de 9 


\section{RESUMEN}

Objetivo: Verificar los conocimientos de los profesionales de la salud sobre principio de atención diferenciada a los pueblos indígenas. Métodos: Se trata de una investigación exploratoria con enfoque cualitativo. Como campo, se eligió un hospital en un municipio del estado de Roraima. Se aplicaron cuestionarios con 11 preguntas semiestructuradas a 7 profesionales de salud. Resultados: La mayoría de los participantes no tenían experiencia de trabajo con pueblos indígenas y tenían un entendimiento limitado, no sabían o no conseguían construir una opinión formada. En el análisis de los discursos de los profesionales, se encontró que conocen algunas características sobre la atención diferenciada, como, por ejemplo, perciben los aspectos culturales y la diversidad lingüística y étnica de los pueblos indígenas. Sin embargo, no hacían relación de estos aspectos, para apoyar actitudes y comportamientos en sus prácticas asistenciales. Conclusión: Ante la percepción de los profesionales de la salud, se evidenciaron diferentes visiones, posibilitando conocer sus vivencias con relación a la realidad del trabajo en el hospital, verificando una preocupación por el conocimiento sobre los pueblos indígenas y con la calidad de la atención. Es necesario revisar la formación de los diferentes profesionales de la salud para prepararlos para el trabajo en contextos interculturales.

Palabras clave: Salud, Pueblos indígenas, Competencia cultural.

\section{INTRODUÇÃO}

Os povos indígenas do Brasil apresentam distintas formas de se relacionar com a sociedade nacional, têm modos de vida peculiares, inclusive em relação ao processo saúde-doença e cuidado (COIMBRA JR. CEA, et al., 2007). No país os indígenas brasileiros estão distribuídos em mais de 250 etnias, apresentam cerca de 180 línguas faladas, com 35 grupos linguísticos (GERSEM LS, 2006). São diferentes, sob a perspectiva de renda, acesso à educação, saúde, trabalho e a situação de exclusão em que vivem (IBGE, 2010; COIMBRA JR. CEA, 2014).

O estado de Roraima apresenta uma população total de 450.479 habitantes divididos entre os 15 municípios: Alto Alegre, Amajarí, Boa Vista, Bonfim, Cantá, Caracaraí, Caroebe, Iracema, Mucajaí, Normandia, Pacaraima, Rorainópolis, São João da Baliza, São Luiz e Uiramutã. Se destaca nacionalmente por haver 49.637 indígenas, que ocupam uma área de 10.370.676 hectares, tendo como os principais povos (etnias): Macuxi, Taurepang, Ingarikó, Yanomami, Y’ekuana, Sanumá, Sapará, Patamona, Wapixana, Waimiri-Atroari e Wai-Wai (RIBEIRO VF, et al., 2020; IBGE CENSO DEMOGRÁFICO, 2010; CARVALHO F, et al., 2008).

A saúde indígena no Brasil é uma temática complexa que vem sendo construída por um histórico caracterizado por conflitos fundiários, acompanhados por uma expansão das fronteiras demográficas nacionais, degradação ambiental, e por vezes, com omissão por parte do Estado. Para os povos indígenas, isso reverbera em retrocessos de avanços sociais verificados no país ao longo das últimas décadas, nos campos da saúde, educação, habitação e saneamento (COIMBRA JR. CEA, 2014).

Em consequência ao expansionismo ocidental, o perfil epidemiológico dos povos indígenas apresenta um histórico de doenças infectocontagiosas, conjuntamente com as infecções sexualmente transmissíveis, doenças crônicas não transmissíveis, como por exemplo, diabetes, hipertensão arterial, obesidade, neoplasias, abuso de álcool e violência (COIMBRA JR. CEA, et al., 2003; MALTA DC, et al., 2017; ALVES APB, et al., 2019).

A Lei 9.836 de 23 de setembro de 1999, estabeleceu o Subsistema de Atenção à Saúde Indígena (SasiSUS) (BRASIL MS, 2019; BRASIL MS, 2009). Esta lei foi determinante para que o governo federal instituísse por meio da portaria $n^{\circ} 254$, de 31 de janeiro de 2002, a Política Nacional de Saúde dos Povos Indígenas (PNASPI) que garante a integralidade da atenção à saúde de forma diferenciada, e que deve ser efetivada em todas níveis de atenção do Sistema Único de Saúde (SUS) (BRASIL MS, 2002).

Ainda com instrumentos legais, como a Constituição Federal de 1988, que reconhece o caráter pluriétnico do país, as Leis Orgânicas de Saúde e a PNASPI, os povos indígenas almejam um SasiSUS realmente diferenciado (BRASIL SF, 2016; BRASIL CNASS, 2003). O princípio da atenção diferenciada procura garantir o respeito as especificidades étincas, epidemiológicas e logísticas desses povos, buscando ofertar serviços 
de saúde efetivos em sua comunidade ou referenciando a outros níveis de complexidade em tempo oportuno, assegurando o respeito a cultura e articulando os saberes tradicionais e os biomédicos (BRASIL MS, 2002).

A definição do conceito de atenção diferenciada ainda está em construção, e a sua prática é um grande desafio (DIEHL EE, et al., 2012). Assim, é fundamental que os profissionais de saúde que irão trabalhar na saúde indígena ou em outro contexto intercultural, estejam preparados, mostrando respeito as especificidades socioculturais, políticas, religiosas, e históricas de cada povo, para poder oferecer em todos os níveis de atenção, serviços de saúde com qualidade aos povos indígenas.

Diante do exposto, este estudo questiona: qual é o olhar dos profissionais de saúde sobre a atenção diferenciada? Deste modo, esta pesquisa tem por objetivo averiguar os conhecimentos de profissionais de saúde sobre o princípio da atenção diferenciada aos povos indígenas.

\section{MÉTODOS}

Trata-se de uma pesquisa exploratória de abordagem qualitativa. Como campo de pesquisa foi escolhido um hospital de um município do estado de Roraima. Os participantes desta pesquisa foram os integrantes da equipe multidisciplinar de saúde que trabalhavam ou estavam fazendo residência no hospital. No total foram sete (7) pessoas que aceitaram participar.

Se utilizou como critério de inclusão no estudo as seguintes condições: ser maior de 18 anos; não ser indígena; ter no mínimo seis meses de experiência de atuação interrupta na instituição, e aceitar de livre e espontânea vontade o convite para particiar da pesquisa. Foram excluídos da pesquisa aqueles que não estivessem em consonância com pelo menos um dos itens acima.

O projeto de pesquisa foi submetido à Plataforma Brasil, e em seguida foi direcionado ao Comitê de Ética e Pesquisa (CEP), e recebeu o parecer favorável com o número: 2.746.119. Os pesquiadores responsáveis e participantes se comprometeram em cumprir com o que dispõem nas normas preconizadas pelo Conselho Nacional de Saúde nas Resolições n 466 de 12 de dezembro de 2012, e n 510 de 7 de abril de 2016, e suas complementares, que tratam dos aspectos éticos das pesquisas que envolvem seres humanos.

A coleta de dados foi relizada a partir de uma entrevista semiestrurada e utilizou-se um questionário com 11 questões abertas e fechadas, sobre a saúde indígena e o conceito de atenção diferenciada. As questões tinham o propósito de favorecer aos participantes a reflexão sobre a importância dos conhecimentos sobre saúde indígena e o atendimento à saúde em contexto intercultural.

Assim, após o convite formal para a participação voluntária e a leitura e assinatura do TCLE, foram realizadas as entrevistas. Todas as entrevistas foram gravadas, com permissão prévia de cada participante, sendo os encontros nos horários de trabalho dos profissionais. Foram entregues, a cada participante 0 questionário, o qual foi primeiramente lido, e caso houvesse alguma dúvida antes ou durante a entrevista, as mesmas foram sanadas imediatamente. A coleta de dados aconteceu no período de julho a agosto de 2018 , dependendo da disponibilidade dos participantes.

Após a efetivação da coleta de dados, os resultados foram transcritos para análise, interpretação e discussão dos achados. As resposta das entrevistas foram submetidas à análise de conteúdo proposta por Minayo MCS (2014), que busca a partir da realização de leituras do conteúdo realizar uma codificação flutuante. Portanto, optou-se por trabalhar com categorias temáticas, que expressam os sentidos que poderão revelar algo de importante sobre o fenômeno estudado.

O estudo não ofereceu nenhum risco previsível e não proporcionou benefícios imediatos a nenhum participante. Mas, espera-se que os resultados provoquem a reflexão, o debate e a motivação de acadêmicos e profissionais de saúde para a importância de buscar conhecimentos sobre o princípio da atenção diferenciada, o que poderá reverberar em uma assistência à saúde efetiva aos povos indígenas.

\section{RESULTADOS}

Após a análise, interpretação e discussão dos dados, emergiram duas categorias temáticas, sendo estas: conhecimento dos profissionais sobre o princípio da atenção diferenciada; importância do conhecimento sobre 
os povos indígenas para o atendimento à saúde hospitalar. Realizou-se primeiramente uma caracterização dos participantes da pesquisa, a partir das seguintes variáveis: idade; sexo; profissão; tempo de atuação no hospital; experiência de trabalho com povos indígenas; e se recebeu capacitação sobre otrabalho em saúde indígena.

O estudo foi composto por 7 profissionais, com idade entre 34 a 48 anos, sendo três do sexo masculino e quatro do sexo feminino. Quanto a profissão, estão representados por: um (1) técnico em enfermagem, uma (1) fonoaudióloga, um (1) fisioterapeuta, um (1) médico, uma (1) assistente social, uma (1) nutricionista, e uma (1) residente em medicina. Em relação ao tempo em que estão atuando no hospial, foi bem diversificado variando de no minímo de seis meses, três anos, cinco anos, sete anos, dez anos, quatorze anos e vinte e dois anos de experiência.

Quanto a possuirem experiência de trabalho com povos indígenas: a maioria afirmou que sim, só dois participantes expressaram que nunca haviam trabalhado com indígenas. A respeito de ter realizado alguma capacitação sobre o trabalho em saúde indígena: a maioria afirmou que nunca realizou. Apenas um participante afirmou ter realizado um curso para atuar de forma direta com a promoção da saúde indígena, em 2017 pela Secretaria Especial de Atenção a Saúde Indígena (SESAI).

\section{Categoria temática I: Conhecimento dos profissionais sobre o princípio da atenção diferenciada}

Esta categoria aborda os significados que são atribuídos pelos profissionais de saúde sobre o princípio da atenção diferenciada aos povos indígenas. Quando questionados sobre se já ouviram falar sobre atenção diferenciada, foram mencionadas diferentes respostas: três disseram qua nunca ouviram falar e quatro responderam que sim, que já haviam escutado sobre. Entretanto, pôde-se perceber que existiam profissionais que não possuíam uma opinião formada, ou não sabiam responder, e os que possuíam uma noção, apresentavam uma visão restrita/equivocada, sobre o conceito de atenção diferenciada, conforme as falas a seguir:

"Acho que por serem do cotidiano, cultura e hábitos peculiares, percebo que a atenção dada a eles seja realmente diferenciada, tento deixá-los o mais próximo possivel da sua realidade" (Entrevistado 1);

"Não sei responder" (Entrevistado 2);

"Ajudar a melhorar a qualidade de atenção em saúde" (Entrevistado 3);

"É quando o indígena tem privilégio no atendimento em relação a outro paciente, em algumas coisas" (Entrevistados 4);

"Não tenho opinião formada" (Entrevistados 5);

"Não sei responder" (Entrevistado 6);

"É o princípio do respeito à diversidade e a dignidade humana" (Entrevistado 7);

Diante das falas, percebeu-se que a maioria apresentava uma visão limitada sobre a atenção diferenciada aos povos indígenas, o que é preocupante pois o hospital em que trabalham é o único de referência do SUS, e os povos indígenas que vivem em Roraima representam mais de $40 \%$ da população do Estado. Os participantes desconhecem o que seria um atendimento diferenciado, e acham que é um privilégio. Vale ressaltar que não é um privilégio, e sim um direito, pelo caráter multicultural do Brasil.

Quando questionados sobre o acompanhamento dos povos indígenas no hospital em relação ao seu entendimento sobre a atenção diferenciada, e se existe, de que forma ocorre? Observou-se os seguintes relatos:

"Acho que existe, em relação a alimentação, ao direito de interprete, a tem local para se colocar redes, caso os indígenas não queiram dormir na cama" (Entrevistado 1);

"Sim, existe mas é precário e sem treinamento" (Entrevistado 2); 
"Sim, existe no hospital um departamento dedicado para os povos indígenas, a coordenação indígena, lá tem interprete" (Entrevistado 3);

"Sim, existe" (Entrevistado 4);

“Não existe" (Entrevistado 5);

"Não tenho essa informação" (Entrevistado 6);

"Sim, existe com uma atenção focada nos costumes de cada região" (Entrevistado 7);

Assim, observa-se que os participantes percebem o seu trabalho dentro de um contexto hospitalar que assiste uma populção diferenciada. Alguns nem conhecem, ou desconhecem em seu próprio atendimento, comportamentos, ações e normativas administrativas institucionais que favorecem a atenção diferenciada para os povos indígenas.

Durante o trabalho hospitalar, o profissional de saúde tem que atender vários pacientes, com vários quadros clínicos, e em diferentes contextos interculturais. Neste sentido, é preciso que estejam constantemente realizando estudos para atender adquadamente seus pacientes. Quando questionados sobre a importância de estarem realizando cursos sobre saúde indígena, os participantes responderam o seguinte:

"Acho que é importante, mas não vejo a instituição promover esses tipos de cursos" (Entrevistado 1);

"Sim, acho importante, sempre em que posso eu faço cursos e participo de capacitações, mas nunca sobre saúde indígena" (Entrevistado 2);

"Sim, faço curso mais na minha área que é de trauma" (Entrevistado 3);

"É importante, mas nunca fiz" (Entrevistado 4);

"Nunca fiz nenhum curso ou capacitação sobre saúde indígena” (Entrevistado 5);

"Sim, já fiz um curso ofertado pela SESAl" (Entrevistado 6);

"Nunca fiz nenhum curso sobre saúde indígena" (Entrevistado 7);

\section{Categoria temática II: Importância do conhecimento sobre os povos indígenas para o atendimento à saúde hospitalar}

Esta categoria versa sobre a percepção e opnião dos profissionais de saúde sobre a assistência em contexto intercultural, e suas experiências de trabalho com os povos indígenas. Diante da percepção dos profissionais de saúde, foram evidenciadas visões diferenciadas, sendo possível a partir dessas conhecer suas experiências em relação a realidade do trabalho no hospital, verificando uma preocupação com os conhecimentos sobre os povos indígenas e com a qualidade na atenção, como ilustra os depoimentos a seguir:

"Sim. Pelo fato de frequentemente estarmos dando assistência a pacientes indígenas, e encontramos dificuldades na comunicação e falta de conhecimento de seus hábitos, principalmente alimentares" (Entrevistado 1);

"Sim. Porque é preciso ter o minímo de conhecimento para atendermos os pacientes indígenas" (Entrevistado 2);

"Sim, é importante devido a barreira cultural e linguística" (Entrevistado 3);

"Sim, é importante porque eles possuem cultura e linguagem diferente da nossa e temos que respeitar" (Entrevistado 4);

"Sim, é importante porque os mesmos fazem parte dos pacientes que são atendidos neste hospital" (Entrevistado 5);

"Sim. Devido às suas necessidades peculiares" (Entrevistado 6); 


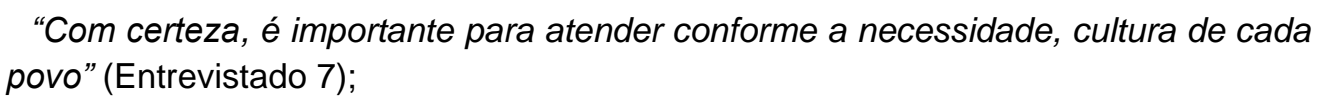

A maioria dos participantes não possuíam experiência de trabalho com povos indígenas, e em relação aos conhecimentos sobre atenção diferenciada apresentavam uma compreensão muito restrita, não sabiam responder ou construir uma opinião sobre o tema. A partir das falas dos profissionais, verificou-se que eles conhecem algumas características sobre atenção diferenciada, como por exemplo, percebem os aspectos culturais e a diversidade linguística e étinica dos povos indígenas. No entanto, não faziam relação desses aspectos, para embasar atitudes e comportamentos em suas práticas assistenciais de saúde.

\section{DISCUSSÃO}

Os sistemas de saúde universais e equânimes, buscam impedir que a identidade étnica e cultural se conceba como um empecilho para acesso integral e oportuno a uma atenção à saúde de qualidade (NORANHA AB, 2010). Os participantes do estudo, percebem a complexidade do trabalho intercultural, seus entendimentos insuficientes apresentam a falta de compreensão sobre os diferentes modos de vida dos povos indígenas do estado de Roraima, exibindo limitações em suas práticas de saúde, por não compreenderem o que seria uma atenção diferenciada.

Assim, é preciso ter conhecimento sobre o que é a interculturalidade. No estudo de Yajahuanca RA, et al. (2015), afirmam que a noção de interculturalidade sugere uma relação de respeito e compreensão no modo de compreender a realidade e o mundo, em um método de informação, educação e formação. Os conceitos sobre "interculturalidade em saúde" ou "saúde intercultural", ultimamente estão sendo utilizados de forma imprecisa para assinalar um anexo de ações e políticas que procuram assumir e incorporar a cultura do paciente no processo de saúde-doença e cuidado.

A interculturalidade apresenta as perspectivas relacional, funcional e crítica. A relacional diz respeito ao contato e ao intercâmbio entre culturas. $\mathrm{O}$ aspecto funcional faz referência ao reconhecimento da diferença e da diversidade cultural com o propósito de sua inclusão na estrutura social estabelecida. Logo, a perspectiva crítica é a mais importante, pois questiona a lógica irracional e instrumental do capitalismo, aponta para o surgimento de novas sociedades, sendo um projeto que busca a transformação das estruturas das relações de poder que mantêm as iniquidades sociais (BRAGATO FF, et al., 2017).

$O$ atendimento diferenciado não é privilégio. Mota SEC e Nunes M (2018) afirmam que os profissionais são resistentes ao SasiSUS, pois acreditam que se trata de "privilégios" aos povos indígenas. Mas, esse pensamento tem analogia com as normativas que organizam o SUS, como o do Decreto ํㅜ 7.508 de 28 de junho de 2011 (BRASIL MS, 2011), que antecipa que "a população indígena contará com regramentos diferenciados de acesso às ações e serviços de saúde, compatíveis com suas especificidades e com a necessidade de assistência integral à sua saúde, de acordo com disposições do Ministério da Saúde".

Para Mota SEC e Nunes M (2018) os textos institucionais devem conter conceitos potentes, claros, e apropriados para explicar os diferentes contextos e culturas em diretrizes para o desenvolvimento de racionalidades relativas a um modo de pensar complexo, sensível e emancipatório. Portanto, existe a necessidade de conceitos e ações para superação da polissemia em torno do princípio da atenção diferenciada, e das distorções em torno da sua aplicabilidade, e ainda auxiliar atuações para o enfrentamento dos processos que mantêm as desigualdades.

Diehl EE, et al. (2012), afirmam que as ofertas de serviços de saúde diferenciados devem considerar o pluralismo e a diversidade cultural, incorporando o direito de participação da comunidade, de forma individual ou coletiva, em seu planejamento, execução e avaliação. Para Yajahuanca RA, et al. (2015), é importante que o respeito conduza o diálogo entre a biomedicina e a medicina tradicional, um conhecimento não pode subjugar o outro, pois causa a quebra do intercâmbio e afeta a interculturalidade.

A PNASPI busca assegurar aos povos indígenas, a integralidade da atenção à saúde, conforme os princípios e as diretrizes do SUS, considerando a diversidade social, cultural, geográfica, histórica e política. 
De modo a apoiar a superação dos fatores que condicionam esses povos a serem mais vulneráveis aos agravos à saúde de maiores proporções e alcance entre a população nacional, aceitando ainda a eficácia de sua medicina e o direito à sua cultura (BRASIL MS, 2002).

A noção de atenção diferenciada para os povos indígenas do Brasil tomou sentido a partir de vários princípios e modelos apresentados em diferentes documentos que fundamentam a história da saúde indígena no país. E esta noção atenta para o "respeito às concepções, valores e práticas em saúde de cada povo e da articulação dos saberes indígenas e biomédicos, (...)" (DIEHL EE, et al., 2012, p.821).

Para execução real da PNASPI, recomenda-se um modelo de atenção organizado de forma diferenciada, tendo como alicerce os Distritos Sanitários organizados em territórios especiais, que respeitam critérios socioculturais, geográficos, históricos e políticos, porém não precisam seguir a mesma divisão geopolítica dos estados e municípios.

A política afirma a condição de vulnerabilidade dos povos indígenas em relação a população nacional, os quais padecem constantemente diante a conflitos, tensão, especialmente em consequência da expansão das frentes econômicas, e a intolerância e ao preconceito, ameaçando sua integridade e organização social (BRASIL MS, 2002).

Diehl EE, et al. (2012) entendem que não existe um conceito claro sobre atenção diferenciada. A atenção diferenciada é referenciada por diferentes noções, como por exemplo, "articulação", "integração" e "incorporação" de práticas tradicionais. As noções de integração e incorporação, sujeitam as práticas tradicionais à análise de eficácia medida pela teoria do conhecimento biomédico. Tal postura se evidencia em um método de organização de serviços de saúde ordenados por um modo capitalista de desenvolvimento, e pela hegemonia do saber biomédico perpetuada nos sistemas de saúde formais.

Nota-se que os profissionais de saúde precisam compreender o sentido da noção de atenção diferenciada, para efetivamente respeitar o que dispõem a PNASPI. Menéndez EL (2003), defende que a compreensão do sentido da palavra "diferenciação" é indispensável para que o profissional de saúde opere em um contexto intercultural, ou seja, em uma perspectiva em que diferentes culturas, visões de mundo, preceitos do processo saúde doença e atenção à saúde estejam acontecendo.

Os profissionais do estudo sabiam pouco ou quase nada sobre os povos indígenas de Roraima, desconheciam a dimensão cultural no processo saúde-doença e cuidado, e por não terem tido experiência teórica e nem prática, torna-se desafiador o trabalho intercultural. Logo, são emergências, na perspectiva intercultural buscar superar desafios em várias áreas. Alguns grupos defendem uma interculturalidade que é oficializada a grupos subalternos, que não tem o propósito de transformação social, em que diferentes grupos possam conviver respeitando os modelos culturais diferenciados (CANDAU VMR e RUSSO K, 2010).

Um caminho possível para o supracitado desafio seria que os profissionais de saúde já na formação vivenciassem a interculturalidade. É evidente que precisamos refletir sobre esses problemas que são pretéritos e precisam ser revistos ainda na universidade, em que os profissionais de saúde amadurecem centralizados pela racionalidade científica e técnica, expressos sobretudo pela dimensão biológica, e é nessa característica que são forjados por meio da autoidentificação profissional com a ciência, com a exclusão das outras formas de cuidado, principalmente aquelas que expressam a dimensão cultural (MENÉNDEZ EL, 2003).

É oportuno enfatizar que o termo interculturalidade surgiu na América Latina, na área da educação, mais precisamente no contexto da educação indígena e no movimento negro latino-americano. Vários estudiosos como por exemplo, Paulo Freire, defendem que as propostas educacionais apresentem diferentes contextos socioculturais e o diálogo entre os diversos saberes (CANDAU VMR e RUSSO K, 2010).

Diehl EE e Pellegrini MA (2014) destacam que a formação superior precisa avançar para colaborar para um desempenho profissional em contextos interétnicos e interculturais. É preciso priorizar as ações de educação permanente em saúde indígena, principalmente aos não-indígenas, ação que precisa ser fortalecida no SasiSUS. Advertem que as experiências em saúde indígena apresentam as limitações da 
formação biomédica para o trabalho intercultural, e estas precisam ser interdisciplinares, que transcendam o campo disciplinar clássico da saúde.

Este estudo apresenta limitações, e não tem a pretensão de definir o conceito de atenção diferenciada, nem de esgotar o tema. Mas, busca fomentar a reflexão e mostrar a necessidade de novos estudos que possam contribuir para os modos de perceber e constituir a diferenciação na atenção em saúde indígena.

\section{CONCLUSÃO}

Diante da percepção dos profissionais de saúde, foram evidenciadas visões diferenciadas, sendo possível conhecer suas experiências em relação a realidade do trabalho no hospital, verificando uma preocupação com os conhecimentos sobre os povos indígenas e com a qualidade na atenção. As formações dos diferentes profissionais de saúde precisam ser revistas, no sentido de prepará-los para o trabalho em contextos interculturais.

\section{AGRADECIMENTOS E FINANCIAMENTO}

Agradecemos aos participantes do estudo, e aos professores e estudantes do Instituto Insikiran de Formação Superior Indígena/UFRR.

\section{REFERÊNCIAS}

1. ALVES APB, et al. Doenças e agravos mais prevalentes em uma comunidade indígena em Boa Vista-RR: relato de experiência. Revista Eletrônica Acervo Saúde, 2019, (26), e673.

2. BRAGATO FF, et al. A interculturalidade como possibilidade para a construção de uma visão de direitos humanos a partir das realidades plurais da américa latina. Revista da Faculdade de Direito. UFPR. Curitiba, 2017; $62(1): 33-59$.

3. BRASIL MS. Saúde indígena: análise da situação de saúde no SasiSUS. Brasília: Ministério da Saúde, $2019 ; 83$ p.

4. BRASIL SF. Constituição da República Federativa do Brasil. Texto constitucional promulgado em 5 de outubro de 1988, com as alterações determinadas pelas Emendas Constitucionais de Revisão nos 1 a 6/94, pelas Emendas Constitucionais nos 1/92 a 91/2016 e pelo Decreto Legislativo no 186/2008. Brasília: Senado Federal, Coordenação de Edições Técnicas, 2016; 496 p.

5. BRASIL. Decreto $n^{\circ} 7.508$, de 28 de junho de 2011. Regulamenta a Lei no 8.080 , de 19 de setembro de 1990 , para dispor sobre a organização do Sistema Único de Saúde - SUS, o planejamento da saúde, a assistência à saúde e a articulação interfederativa, e dá outras providências. Diário Oficial da União, Brasília, DF, 29 jun. 2011. Seção 1, p. 1.

6. BRASIL MS. Fundação Nacional de Saúde. Lei Arouca: a Funasa nos 10 anos de saúde indígena. Brasília: Funasa, 2009; 112 p.; il.

7. BRASIL CNASS. Legislação do SUS. Brasília: CONASS, 2003; 604 p.

8. BRASIL MS. Fundação Nacional de Saúde. Política Nacional de Atenção à Saúde dos Povos Indígenas. $2^{\mathfrak{a}}$ edição. Brasília: Ministério da Saúde, 2002; 40 p.

9. CANDAU VMR, RUSSO K. Interculturalidade e educação na américa latina: uma construção plural, original e complexa. Rev. Diálogo Educ., Curitiba, 2010; 10(29): 151-169.

10. CARVALHO F, et al. História, Cultura e Meio Ambiente em Roraima: perspectivas interculturais. Editora da Universidade Federal de Roraima. Boa Vista-RR. 2008; 90 p.

11. COIMBRA JR. CEA. Saúde e povos indígenas no Brasil: reflexões a partir do I Inquérito Nacional de Saúde e Nutrição Indígena. Cad. Saúde Pública, Rio de Janeiro, 2014; 30(4): 855-859.

12. COIMBRA JR. CEA, et al. Processo saúde-doença. In: BARROS DC, SILVA, et al (organ.). Vigilância alimentar e nutricional para a saúde Indígena [online]. Vol. 1. Rio de Janeiro: Editora FIOCRUZ, 2007; pp. 47-74.

13. COIMBRA JR. CEA, et al. (organ.). Epidemiologia e Saúde dos Povos Indígenas no Brasil. Rio de Janeiro: Fiocruz/Abrasco; 2003; 13-47: 2327-2339.

14. DIEHL EE, et al. Contribuição dos agentes indígenas de saúde na atenção diferenciada à saúde dos povos indígenas brasileiros. Cad. Saúde Pública, Rio de Janeiro, 28 (5): 819-831, mai., 2012.

15. DIEHL EE, PELLEGRINI MA. Saúde e povos indígenas no Brasil: o desafio da formação e educação permanente de trabalhadores para atuação em contextos interculturais. Cad. Saúde Pública, Rio de Janeiro, 2014; 30(4): 867-874.

16. GERSEM LS. O Índio Brasileiro: o que você precisa saber sobre os povos indígenas no Brasil de hoje. Brasília: Ministério da Educação, Secretaria de Educação Continuada, Alfabetização e Diversidade; LACED/Museu Nacional, 2006.

17. IBGE. INSTITUTO BRASILEIRO DE GEOGRAFIA E ESTATÍSTICA. CENSO DEMOGRÁFICO 2010. Características da população e dos domicílios. Resultados do Universo. Disponível em: https://biblioteca.ibge.gov.br/visualizacao/periodicos/93/cd_2010_caracteristicas_populacao_domicilios.pdf Acesso em: 07/07/2020.

18. MALTA DC, et al. Doenças crônicas não transmissíveis e a utilização de serviços de saúde: análise da Pesquisa Nacional de Saúde no Brasil. Rev. Saúde Pública. 2017; 51 Supl 1:4s. 
19. MENÉNDEZ EL. Modelos de atención de los padecimentos: de exclusiones teóricas y articulaciones práticas. Ciência \& Saúde Coletiva, Rio de Janeiro, 2003; 8(1): 185-207.

20. MINAYO MCS. O Desafio do Conhecimento: pesquisa qualitativa em saúde. São Paulo; Hucitec; $2014 ; 14: 407$.

21. MOTA SEC, NUNES M. Por uma atenção diferenciada e menos desigual: o caso do Distrito Sanitário Especial Indígena da Bahia. Saúde Soc. São Paulo, 2018; 27(1): 11-25.

22. NORONHA AB. Interculturalidade em saúde: formando para o diálogo. Revista RETES, Ano 2 - n 6 - abri. /mai. /jun., 2010.

23. RIBEIRO VF, et al. Estudo epidemiológico sobre o vírus da imunodeficiência humana (HIV) em indígenas do estado de Roraima entre 2010 a 2018. Revista Eletrônica Acervo Saúde, 12(7), e3447.

24. YAJAHUANCA RA, et al. É preciso "ikarar os kutipados": interculturalidade e assistência à saúde na Amazônia Peruana. Ciência \& Saúde Coletiva, 20(9):2837-2846, 2015. 